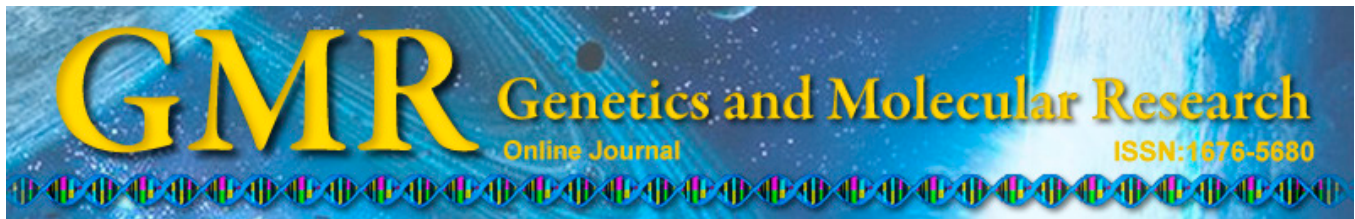

\title{
Relationship between abnormal NOS expression and the pathogenesis of cerebral aneurysm
}

\author{
W.S. Liu ${ }^{1,2}$, H.J. Geng ${ }^{3}$, C.D. Wang ${ }^{4}$, A.J. Li ${ }^{2}$, P.C. Cao ${ }^{2}$, D.K. Wang ${ }^{2}$ and G. Li \\ ${ }^{1}$ Department of Neurosurgery, Qi Lu Hospital, Shandong University, Jinan, China \\ ${ }^{2}$ Department of Neurosurgery, Weifang People's Hospital, Shandong, China \\ ${ }^{3}$ Department of Clinical Laboratory, \\ Affiliated Hospital of Weifang Medical College, Weifang, China \\ ${ }^{4}$ Centre Laboratory, Weifang People's Hospital, Shandong, China
}

Corresponding author: G. Li

E-mail: doctorgangli88@163.com

Genet. Mol. Res. 14 (2): 4276-4281 (2015)

Received June 30, 2014

Accepted October 20, 2014

Published April 28, 2015

DOI http://dx.doi.org/10.4238/2015.April.28.9

\begin{abstract}
We sought to investigate the relationship between abnormal expression of nitric oxide synthase (NOS) and pathogenesis of cerebral aneurysm. Brain tissues were collected from 36 patients with cerebral aneurysm confirmed by computer tomography with angiography or neurosurgical therapy. The control group consisted of 25 patients of similar age who had no vascular diseases, as confirmed by magnetic resonance imaging. Samples of cortical arterioles were collected. The structure of the aneurysms was detected by hematoxylin and eosin staining, and the expression of inducible NOS was detected by immunohistochemistry. NOS expression was significantly higher in the patient group than in the control group (patients: $30 / 36$ strongly positive; control: $0 / 25$ strongly positive; $\mathrm{P}<0.05$ ). In conclusion, the pathogenesis underlying cerebral aneurysm may be due to abnormal expression of NOS, degradation of the extracellular matrix, aggravation of a pro-inflammatory reaction, or a deficiency in arterial elasticity
\end{abstract}


layer synthesis. These changes may result in a deficiency in vascular remodeling.

Key words: NOS; Cerebral aneurysm; Immunohistochemistry

\section{INTRODUCTION}

Cerebral aneurysm is a kind of neurovascular disorder that occurs in the branches of cerebral arteries. If an aneurysm ruptures, it causes a subarachnoid hemorrhage, followed by cerebral thrombosis, and hypertensive cerebral hemorrhage. Cerebral aneurysms have high recurrence and cause mortality, which can seriously affect the health of the patients.

Cerebral aneurysms could be roughly divided into different groups according to the location of the tumors, namely, internal carotid artery, anterior cerebral artery, middle cerebral artery, basilar artery, or giant aneurysms (Schwenk et al., 2010; Michel et al., 2011). Previous studies have reported that aneurysms that occur in the branches of the internal carotid artery account for $40 \%$ of all the cerebral aneurysms, with the most common site being branches of the posterior communicating artery. Aneurysms that occur in the branches of the anterior cerebral artery, including the anterior communicating artery, account for $30 \%$, and those in the middle cerebral artery account for $20 \%$, with the most common site being the trigeminal part. Aneurysms that occur in peripheral part of anterior cerebral and vertebral artery both account for $5 \%$ of all aneurysms. Multiple aneurysms often occur in branches of the middle cerebral artery, posterior communicating artery of the internal carotid artery, and ophthalmic artery (Golledge et al., 2010; Martinez-Pinna et al., 2010).

Recently, there has been progress in the etiology of cerebral aneurysms; however, their pathogenesis is yet to be elucidated. At present, the change in hemodynamics and inflammatory response of the extracellular matrix, caused by atherosclerosis, are considered crucial in the occurrence of cerebral aneurysms. Some reports have indicated that high expression of nitric oxide synthase (NOS), mainly of inducible nitric oxide synthase (iNOS), due to external factors result in an increase in nitric oxide (NO). This could cause degradation of the extracellular matrix and carcinogenesis of cells (Seidel et al., 2010; van Kuijk et al., 2010; Ramos-Mozo et al., 2012). In this study, we compared the expression of iNOS in tissue from various cerebral aneurysms to illustrate the abnormal expression of iNOS in the pathogenesis of cerebral aneurysms, and provide data for further research on the role of NOS in the pathogenesis of tumors.

\section{MATERIAL AND METHODS}

\section{General information}

The data from 36 patients from Qilu Hospital of Shandong University were collected and analyzed. All patients were diagnosed with cerebral aneurysm by neurosurgical therapy or computer tomography with angiography (CTA). The patient group included 12 cases of anterior communicating artery aneurysm, 11 cases of posterior communicating artery aneurysm, 7 cases of middle cerebral artery aneurysm, and 6 cases of internal carotid artery aneurysm. Twenty cases were males and 16 were females. The average age of the patients was 48.35 years (ranging from 17-62 years). The tumor tissue was collected by surgical operation. The 
control group consisted of 25 patients of similar ages and without vascular disease. All the patients in the control group had no vascular diseases, history of hypertension, hyperlipidemia, diabetes, or family history of vascular cancer. The cortex arteriole was collected from all patients of both groups. The structure of the aneurysm was detected by hematoxylin and eosin (H\&E) staining and the expression of NOS was detected by immunohistochemistry. The protocol of this study was approved by the Qilu Hospital of Shandong University (Shandong, China). Informed consent was obtained from all subjects.

\section{Reagents}

Tissue lysis buffer was bought from Tiangen Biology Corporation. The antibodies were acquired from Santa Cruz Biotechnology. The reagents for H\&E staining were bought from Dingguo Biology Corporation. The kit to detect the bioactivity of NOS was purchased from Roche.

\section{CTA detection}

All the patients received CTA examination to detect the location of the cerebral aneurysm and facilitate the operation. The CTA examination was conducted by the Radiology Department and they provided the data for analysis.

\section{Preparation of pathological slides and analysis}

The expression of NOS was detected by immunohistochemistry. The tissue slides were prepared in the Department of Pathology. They were fixed in $10 \%$ formalin and then dehydrated and paraffin-embedded. The slides were stained with H\&E for 10 min and then dehydrated, cleared, sealed, and observed with a microscope. They were assessed using a double-score semi-quantitative integral method according to the number of stained cells and the strength of the dye. The scoring scheme for the number of positive cells was: $0=<5 \%, 1$ $=6-25 \%, 2=26-50 \%, 3=51-75 \%, 4=>75 \%$. The scoring scheme for the strength of the dye was: $0=$ no dye, $1=$ sundown, $2=$ claybank, and $3=$ sepia. The total score was determined by the sum of both. A score of 0 was negative (-), 1-4 was weakly positive (+), 5-8 was positive $(++),>9$ was strongly positive $(+++)$.

\section{Assessing NOS expression by immunohistochemistry}

The expression of NOS was detected using the streptomycin-avidin-biotin-peroxidase complex (SABC) method. The procedure was performed following the routine method for SABC staining. The primary antibody dilution was 1:100.

\section{Statistical analysis}

Data are reported as means \pm SD. Statistical comparison between groups was performed using an independent samples $t$-test. All data were analyzed in SPSS 11.0. The significance was set at $\mathrm{P}<0.05$. 


\section{RESULTS}

\section{Results of CTA}

All patients received CTA examination, which showed the location and size of the tumor very clearly. Some of these results are shown in Figure 1.
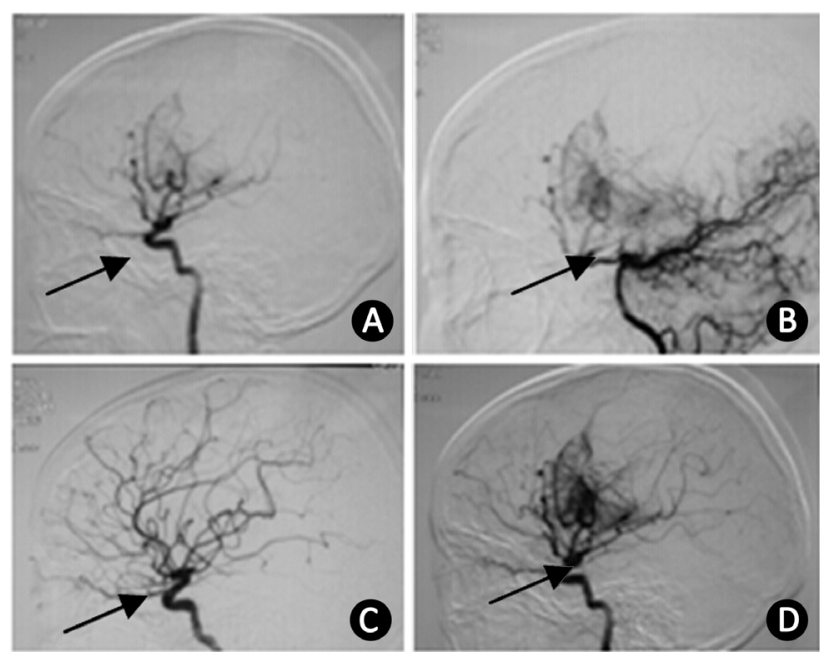

Figure 1. Representative radiographic images of an aneurysm. A., B., C., D. show the location of aneurysms in some patients.

\section{Assessment of pathological changes in cerebral aneurysm}

The results of H\&E stain showed that significant changes occurred in the artery wall, in particular in the intima and tunica media, of the aneurysms; the typical three-layer structure disappeared. Fibrous thickening occurred in the intima, the cells in the intima reduced, and their continuity was interrupted. Muscular atrophy occurred in the tunica media, while infiltration of foam cells and lymphocytes significantly increased. The structure of the artery in the control group was normal. The endothelial cells were complete and uniform. The smooth muscle fibers in the tunica media were well-ordered and densely arranged, and no inflammation was detected. There was a significant increase in the score for NOS-positive cells and strength of dye in the patients compared with the control group $(\mathrm{P}<0.05)$. The results indicated that the expression of NOS was significantly increased in the pathogenesis of cerebral aneurysms (Table 1).

Table 1. Scores for NOS expression in the two groups.

\begin{tabular}{|c|c|c|c|c|}
\hline & - & + & ++ & +++ \\
\hline Patients $(\mathrm{N}=36)$ & 0 & 0 & 6 & 30 \\
\hline Controls $(\mathrm{N}=25)$ & 23 & 2 & 0 & 0 \\
\hline$t$ value & 4.568 & 4.698 & 3.982 & 3.236 \\
\hline$P$ value & $<0.05$ & $<0.05$ & $<0.05$ & $<0.05$ \\
\hline
\end{tabular}

$\mathrm{P}<0.05$ versus the control group. 


\section{Identification of the expression of NOS}

The expression of total NOS between the two groups was statistically comparable; however, the expression of iNOS significantly increased in the patient group compared with the control group (Table 2; $\mathrm{P}<0.05$ ).

\begin{tabular}{lcr}
\multicolumn{2}{c}{ Table 2. NOS expression in the two groups (mean $\pm \mathrm{SD}, \mathrm{U} / \mathrm{mg}$ protein). } & iNOS \\
\hline & Total NOS & $13.3129 \pm 2.4581$ \\
\hline Patient group & $24.5631 \pm 6.5684$ & $7.5623 \pm 2.0321$ \\
Control group & $22.1325 \pm 6.1358$ & 4.967 \\
$t$ value & 1.230 & $0.032^{*}$ \\
$\mathrm{P}$ value & 0.562 &
\end{tabular}

\section{DISCUSSION}

Congenital deficiency of the cerebral artery wall and increase in the intraluminal tension result in a thin artery wall and an abnormal projection, forming a cerebral aneurysm. Cerebral aneurysms are mainly located in the bifurcation of cerebral artery and branches of the main arteries because these locations are susceptible to congenital deficiencies of the muscular layer and endure the strongest impact of blood flow. When subjected to long-term tension of blood flow, the weak point of the artery wall becomes projected and dilated, forming a cerebral aneurysm (O'Hare et al., 2010). At present, the management of cerebral aneurysms includes medical and surgical treatment. Medical treatment is mainly used to treat un-ruptured aneurysms by relieving the stimulation to the aneurysm wall through controlling the risk factors associated with the pathogenesis of cerebral aneurysms (Chan et al., 2013). Surgical treatment is a direct action on the cerebral aneurysm through medical devices, including clipping or ligation of the aneurysm neck, isolation of the aneurysm, and packing of the aneurysm wall. However, the etiology and progression of cerebral aneurysms are currently unclear. Many studies have indicated that the pathogenesis of cerebral aneurysms is related to changes in the expression of cytokines, such as nerve growth factor, transforming growth factor $\beta 1$, specificity protein 1, and matrix metalloproteinases (Hughes et al., 2005; Hoare et al., 2011; Wojtkowiak et al., 2011; Efthimiadou et al., 2014). However, this has not been confirmed; therefore, the mechanism of the pathogenesis of cerebral aneurysm is yet to be elucidated.

Cerebral aneurysms could rupture at any time, depending on their specific location and characteristics. Psychentonia, emotional excitement, strain, or vigorous head exercise could result in a sudden rise in the blood pressure, causing the aneurysm to rupture (Bock et al., 2010). In the present study, CTA was used to clearly locate cerebral aneurysms.

NO is a free radical that produces biological effects through reactions with superoxide and sulfhydryl compounds. It plays a crucial role in the physiology and pathology of the body. There are two forms of NOS in the body: constitutive NOS and iNOS. Constitutive NOS is a calcium ion-dependent enzyme, which is important in nervous signal transduction and has a short activity time. iNOS is a calcium ion-independent regulatory enzyme, which has a longer active time, increases under pathological conditions and is active in the process of cell death and carcinogenesis (Hughes et al., 2005; Bock et al., 2010). Some studies have indicated no expression of NOS in endothelial cells and muscular cells under normal conditions; however, increased expression is seen under pathological conditions, resulting in a local increase of NO 
that may induce cell death and carcinogenesis (Seidel et al., 2010; Hoare et al., 2011). In the present study, we studied the relationship between the expression of iNOS and pathogenesis of cerebral aneurysm. Our results indicated that the expression of NOS significantly increased in patients with cerebral aneurysms when compared with the control group. We speculated that NOS plays a role in the pathogenesis of cerebral aneurysms.

We focused on NOS because it is closely associated with tumors. The expression of NOS was detected in the cerebral aneurysms, indicating that the expression of NOS was increased in the pathogenesis of cerebral aneurysms. The results were similar to previous studies that suggest that the expression of NOS is increased in tumors; however, further research is needed to assess whether the overexpression of NOS would increase the ability of tumor cell proliferation and migration through the expression of its cell surface receptor.

\section{Conflicts of interest}

The authors declare no conflict of interest.

\section{REFERENCES}

Bock N, Riminucci A, Dionigi C, Russo A, et al. (2010). A novel route in bone tissue engineering: magnetic biomimetic scaffolds. Acta Biomater. 6: 786-796.

Chan A, Orme RP, Fricker RA and Roach P (2013). Remote and local control of stimuli responsive materials for therapeutic applications. Adv. Drug. Deliv. Rev. 65: 497-514.

Efthimiadou EK, Tapeinos C, Tziveleka LA, Boukos N, et al. (2014). pH- and thermo-responsive microcontainers as potential drug delivery systems: Morphological characteristic, release and cytotoxicity studies. Mater. Sci. Eng. C Mater. Biol. Appl. 37: 271-277.

Golledge J, Clancy P, Moran C, Biros E, et al. (2010). The novel association of the chemokine CCL22 with abdominal aortic aneurysm. Am. J. Pathol. 176: 2098-2106.

Hoare T, Timko BP, Santamaria J, Goya GF, et al. (2011). Magnetically triggered nanocomposite membranes: a versatile platform for triggered drug release. Nano Lett. 11: 1395-1400.

Hughes S, El Haj AJ and Dobson J (2005). Magnetic micro- and nanoparticle mediated activation of mechanosensitive ion channels. Med. Eng. Phys. 27: 754-762.

Martinez-Pinna R, Barbas C, Blanco-Colio LM, Tunon J, et al. (2010). Proteomic and metabolomic profiles in atherothrombotic vascular disease. Curr. Atheroscler. Rep. 12: 202-208.

Michel JB, Martin-Ventura JL, Egido J, Sakalihasan N, et al. (2011). Novel aspects of the pathogenesis of aneurysms of the abdominal aorta in humans. Cardiovasc. Res. 90: 18-27.

O’Hare AM, Fanning NF, Ti JP, Dunne R, et al. (2010). HydroCoils, occlusion rates, and outcomes: a large single-center study. AJNR. Am. J. Neuroradiol. 31: 1917-1922.

Ramos-Mozo P, Rodriguez C, Pastor-Vargas C, Blanco-Colio LM, et al. (2012). Plasma profiling by a protein array approach identifies IGFBP-1 as a novel biomarker of abdominal aortic aneurysm. Atherosclerosis 221: 544-550.

Schwenk JM, Igel U, Kato BS, Nicholson G, et al. (2010). Comparative protein profiling of serum and plasma using an antibody suspension bead array approach. Proteomics 10: 532-540.

Seidel MF, Herguijuela M, ForkertR and Otten U (2010). Nerve growth factor in rheumatic diseases. Semin. Arthritis Rheum. 40: 109-126.

van Kuijk JP, Flu WJ, Chonchol M, Bax JJ, et al. (2010). Metabolic syndrome is an independent predictor of cardiovascular events in high-risk patients with occlusive and aneurysmatic peripheral arterial disease. Atherosclerosis 210: 596-601.

Wojtkowiak JW, Verduzco D, Schramm KJ and Gillies RJ (2011). Drug resistance and cellular adaptation to tumor acidic pH microenvironment. Mol. Pharm. 8: 2032-2038. 